they need to collaborate with scholars of society-at-large. Sociologists and philosophers of science, in turn, are acquiring a more intimate understanding of the scientists that they study.

These promising developments are being driven by a wider political context. In the United States, the events of 11 September 2001 and the subsequent wars in Afghanistan and Iraq led to a reassessment of the role of social-sciences research, particularly in regard to its relevance to national security. This led to firmer, bipartisan support for the social, behavioural and economic sciences directorate at the National Science Foundation. In Europe, meanwhile, strong public suspicion of new technologies - which has had particularly devastating consequences for the deployment of genetically modified crops in Europe and beyond - has encouraged governments to set aside more resources for the early involvement of social scientists in technology development.

In this issue, we report on the strengths and weaknesses of a UK initiative to bring the social sciences to bear more effectively on genomics, and on the life sciences more generally (see page 840 ). This experiment suggests that coherent, multidisciplinary centres can help social scientists to get a firmer grip on the complex science, cultures and behaviours underlying new technologies. But it also highlights the need for funding agencies, such as the UK Economic and Social Research Council, to retain a close interest in the strategic direction of such centres, and to ensure that their successes and failures are noted and built upon, even after their direct funding has expired.

The increased involvement of social scientists in science and technology issues has been especially pronounced of late in nascent fields such as nanotechnology and synthetic biology, where funding agencies feel that they have to tread carefully lest their work unleashes a backlash from the public.

In these areas, it is too early to assess the value - and beneficiaries - of the social scientists' contribution. The idea of embedding sociology, law and philosophy firmly in the development of a scientific discipline from the outset is only now being tested. There is optimism among many of the engineers and natural and social scientists involved.

However, all the signs are that the various parties are approaching these collaborations very much on their own terms. Natural scientists are under pressure to deliver new insight and applications. As far as they are concerned, if social scientists wish to observe them, that's probably tolerable.

Social scientists, in turn, wish to be respected for their insight into how scientists and their ideas function both within their communities and, above all, in relation to societal ambitions and values. These researchers do not, by and large, see their purpose as being to pre-empt societal reactions or public engagement, or to help natural scientists communicate. Moreover, for them, the tension between collaboration and detachment in these projects is real.

There is a possibility, therefore, that these parties will end up walking and talking past each other. What is more, the management of rigorous programmes involving both groups is
"Coherent, multidisciplinary centres can help social scientists to get a firmer grip on the complex science, cultures and behaviours underlying new technologies." hampered by the difficulty that the social scientists (and those who support them) have in reaching agreement on what constitutes outstanding analysis of human practices in these contexts: just how that can best be achieved, and to what extent, should be at the service of government policy goals.

None of this should encourage a dismissive attitude among sceptics. The applications of genetics, nanotechnology, synthetic biology and other technologies are giving rise to substantial new challenges in professional practice and communication, in ethics, in intellectual property and in many other dimensions beyond the science itself. Objective insights into these dimensions have their own value, and the new collaborations should help. The challenge remains to identify how that value can best be fulfilled.

\section{A class of their own}

\section{The Japanese winners of Nature's mentoring awards have the universal qualities of outstanding advisers.}

$1 / \square$ $\mathrm{r}$ Kitano is always ready to invest in apparently absurd ideas.... He actively seeks to gain international exposure for his young researchers by making them corresponding authors on his papers.... His distinctive mentoring style - decisiveness and respect for the individual - comes from the fact that he is not a pure product of the Japanese system."

"The thing that most surprised me when I joined the Oosawa group was that everybody called him 'Oosawa-san' [rather than the much more formal 'Oosawa-sensei']. Also, rather than sitting in an office ... he walked around the lab collaring people and talking with them."

These two extracts are drawn from the enthusiastic nominations of Hiroaki Kitano, head of Sony Computer Science Laboratories in Tokyo, and Fumio Oosawa, a biophysicist at Aichi Institute of Technology in Toyota - the respective winners of the 2009 ' $\mathrm{mid}$ career' and 'lifetime achievement' awards given by Nature for scientific mentoring. Since the awards' inception in 2005, they have been held in a different country every year, and they have been judged each time by a multidisciplinary panel of leading scientists from that country (see go.nature.com/Rccbo4).

An account of this year's awards, which took place in Japan, can be found on page 948. As in previous years, the two winners display accessibility, a broad and insightful overview, and an ability to engage with young researchers on the latter's own terms - qualities that seem to be common to outstanding mentors everywhere. There is no doubt that the Japanese system tends to be strongly hierarchical, but it was clear to the judges that, as in all other countries in which the competition has been held, qualities that buck such hierarchies lead to outstanding new generations. Congratulations to Oosawa and Kitano. 